\title{
VARIACIÓN FENOTÍPICA ENTRE Y DENTRO DE POBLACIONES SILVESTRES DE CHILE DEL NOROESTE DE MÉXICO
}

\author{
PHENOTYPIC VARIATION AMONG AND WITHIN WILD POPULATIONS OF PEPPER FROM \\ NORTHWESTERN MÉXICO
}

\author{
Sergio Hernández Verdugo ${ }^{*}$, Ricardo G. López España ${ }^{1}$, Pedro Sánchez Peña ${ }^{1}$, \\ Manuel Villarreal Romero ${ }^{1}$, Saúl Parra Terraza ${ }^{1}$, Flor Porras ${ }^{2}$ y José L. Corrales Madrid ${ }^{1}$
}

\begin{abstract}
${ }^{1}$ Facultad de Agronomía, Universidad Autónoma de Sinaloa. Km 17.5 Carretera Culiacán-El Dorado. Apdo. Postal 726. 80000, Culiacán Sinaloa, México. Tel. y Fax 01 (667) 846-1084. ${ }^{2}$ Laboratorio de Inmunología, Departamento de Bioquímica, Facultad de Medicina, Universidad Nacional Autónoma de México.
\end{abstract}

*Autor para correspondencia (sergioh2002mx@yahoo.com.mx)

\section{RESUMEN}

Se estimó la distribución jerárquica de la variación fenotípica entre poblaciones, entre y dentro de familias, y la proporción de esta variación que tiene una base genética en cuatro poblaciones de chile silvestre (Capsicum annuum var. glabriusculum) del noroeste de México. Los caracteres medidos fueron: altura de planta, diámetro de tallo, largo de hoja, ancho de hoja, número de frutos por planta, número de semillas por fruto, número de semillas por planta y peso de semilla. De la variación total, $61.8 \%$ se distribuyó dentro de familias, $24.7 \%$ entre familias y $\mathbf{1 3 . 5} \%$ entre poblaciones. De la variación fenotípica, total $38.2 \%$ tiene una base genética, de la cual $30.1 \%$ se distribuyó entre y $69.9 \%$ dentro (de familias) de poblaciones. La heredabilidad en sentido amplio varió entre características y entre poblaciones; en altura de planta, diámetro de tallo, largo de hoja, número de frutos por planta, número de semillas por planta y peso individual de semilla, la heredabilidad fue mínima. En ancho de hoja y número de semillas por fruto, los valores de heredabilidad variaron de 0.10 hasta 0.75 . En tres poblaciones los valores de heredabilidad mínimos fueron cero o cercanos a cero en tres características diferentes en cada población, mientras que en la población restante variaron de 0.23 a 0.75 . Las dos poblaciones adyacentes (Yecorato Mezquite y Yecorato Camino) separadas por sólo $500 \mathrm{~m}$ de distancia difirieron sustancialmente en la heredabilidad de cinco de las ocho características analizadas. Estos resultados sugieren que el potencial para el cambio evolutivo de una característica particular varía ampliamente entre las poblaciones estudiadas, aún en aquéllas separadas por pocos metros. La variación dentro de familias fue alta y se distribuyó uniformemente a través de las poblaciones en todas las características, excepto en diámetro de tallo. Esta alta variación puede ser causada por diferencias en la plasticidad de los individuos dentro de las familias.

Palabras clave: Capsicum annuum silvestre, variación genética, heredabilidad.

\section{SUMMARY}

The hierarchical distribution of phenotypic variation among populations, among and within families, and the proportion of such variation genetically based was estimated in four populations of wild pepper (Capsicum annuum var. glabriusculum) from northwestern México. The traits measured were: plant height, stem diameter, leaf length, leaf width, number of fruits per plant, number of seeds per plant, number of seeds per fruit and seed weight. Out of the total variation, $61.8 \%$ was found within families, $24.7 \%$ among families and $13.5 \%$ among populations. From the total phenotypic variation, $38.2 \%$ was genetically based, from which $30.1 \%$ was among and $69.9 \%$ within (in families) populations. The broad sense heritability varied among traits and populations. Plant height, stem diameter, leaf length, number of fruits per plant, number of seeds per plant and individual seed weight showed minimum heritability values. In leaf width and number of seeds per fruit, the heritability values varied from 0.10 to 0.75 . In three populations the minimum values of heritability were of zero or close to zero for three different traits in each population, while in the remaining populations the heritability values varied from 0.23 to 0.75 . The two adjacent populations (Yecorato Mezquite and Yecorato Camino) separated by only $500 \mathrm{~m}$ differed substantially in the heritability of five out of eight traits analyzed. These results suggest that the potential for evolutionary change in a particular trait varies widely among the studied populations, even in those populations separated by a small distance. Within family variation was high and uniformly distributed across populations in all traits, except in stem diameter. This high variation can be caused by differences in plasticity of individuals within families.

Index words: Capsicum annuиm wild type, genetic variation, heritability.

\section{INTRODUCCIÓN}

El potencial evolutivo de las especies que viven en condiciones naturales depende de sus niveles de variación genética y la manera de cómo se distribuye esta variación dentro y entre sus poblaciones (Hamrick y Godt, 1997). Una población o especie con bajos niveles de variación genética puede sobrevivir y mantenerse en un ambiente determinado, pero corre el riesgo de extinguirse cuando estas condiciones se modifican. Se considera que el 
cambio evolutivo es conducido principalmente por los procesos de selección natural y la deriva génica al azar (Wright, 1951). La selección natural opera en aquellas poblaciones donde sus individuos presentan diferencias entre sí, y cuando tales diferencias son heredables y tienen un valor adaptativo; en cambio, la deriva génica juega un papel importante en las poblaciones de tamaño pequeño (Kimura y Otha, 1971).

La fragmentación y destrucción del hábitat donde se encuentran las poblaciones silvestres parientes de las plantas cultivadas, hace que el estudio y estimación de los niveles de variación genética y su distribución entre y dentro de las poblaciones sean aspectos necesarios para su manejo y conservación (Vida, 1994). Existen varios métodos usados ampliamente para estimar los niveles de diversidad genética, tales como isoenzimas, marcadores RAPD's y microsatélites. Sin embargo, el estudio de caracteres fenotípicos es uno de los primeros pasos para caracterizar el germoplasma que se encuentra en condiciones naturales en México.

Diversos investigadores han indicado que la distribución de la variación fenotípica entre y dentro de las poblaciones, así como la proporción heredable de ésta, dependen del carácter y la especie estudiadas (Rice y Mack,1991; Cahill y Ehdaie, 2005; Felde et al., 2006; Zhang y Zhou, 2006). En un estudio efectuado en invernadero, Rice y Mack (1991) reportaron que en Bromus tectorom la mayor parte de la variación en casi todos los caracteres fenotípicos medidos se distribuyó principalmente dentro de familias; debido a que esta especie se reproduce exclusivamente por autofecundación, Rice y Mack (1991) concluyeron que la alta variación observada dentro de familias se debía a la presencia de un componente significativo de plasticidad entre individuos. En cambio, Cahill y Ehdaie (2005) encontraron que la mayor parte de la variación fenotípica medida entre accesiones silvestres y cultivadas de Salvia hispanica, tiene una base genética y es heredable. Felde et al. (2006) y Zhang y Zhou (2006) reportaron resultados similares para poblaciones de Brassica napus.

El género Capsicum (Solanaceae) está formado por alrededor de 30 especies (Hernández-Verdugo et al., 1999), de las cuales $C$. annuum, $C$. chinense, $C$. frutescens, $C$. baccatum y $C$. pubescens son domesticadas. De las domesticadas, C. annuum es la especie de mayor importancia económica, se cultiva ampliamente en todo el mundo y se considera que fue domesticada en México (Pickersgill, 1971a). Esta especie es la que presenta la mayor variación en tamaño, color y forma de los frutos. A ella pertenecen los tipos de chiles conocidos como 'Serrano', 'Jalapeño', 'Ancho' y 'Morrón' o 'Bell pepper', entre otros. Las plantas de chile silvestre (Capsicum annuum var. glabriusculum (Dunal) Heiser y Pickersgill), conocidas comúnmente como chiles "chiltepines" o "piquines" son perennes, herbáceas o trepadoras, sus frutos son pequeños, rojos y picantes; son comidos por las aves que dispersan sus semillas (Vázquez-Dávila, 1996). Las poblaciones de chile silvestre se distribuyen ampliamente por todo el territorio nacional. Es posible encontrarlas en sitios imperturbados de la selva baja caducifolia, así como a orillas de los caminos, en huertos, potreros y bajo la vegetación remanente a orillas de los campos de cultivo (HernándezVerdugo et al., 1999), generalmente en altitudes menores de 1000 m (D' Arcy y Eshbaugh, 1974).

Estudios recientes con isoenzimas y marcadores moleculares RAPD's (Hernández-Verdugo et al., 2001a, 2006; Oyama et al., 2006) indican que las poblaciones de chile silvestre del noroeste de México mantienen altos niveles de variación genética dentro y entre sus poblaciones. Estas poblaciones también variaron significativamente en capacidad de germinación de sus semillas y en resistencia contra el geminivirus PHV (Hernández-Verdugo et al., 2001b y c). Sin embargo, se desconoce la cantidad de la variación en caracteres cuantitativos y su distribución entre y dentro de las poblaciones. En este estudio se analizaron poblaciones de $C$. annuum silvestre del noroeste de México con el objetivo de determinar la distribución de la variación fenotípica entre poblaciones, entre y dentro de familias, y estimar la proporción de esta variación fenotípica que tiene una base genética.

\section{MATERIALES Y MÉTODOS}

Material vegetal y diseño experimental. Se colectaron frutos maduros de cuatro poblaciones de chiles silvestres en el Estado de Sinaloa, distribuidas en una distancia de aproximadamente $250 \mathrm{~km}$, entre noviembre y diciembre de 2005. Las poblaciones Yecorato Mezquite y Yecorato Camino estaban separadas por una distancia de 500 m. La población Texcalama se localizó a $120 \mathrm{~km}$ de las poblaciones anteriores, y la población Alcoyonqui a 130 $\mathrm{km}$ de esta última.

Dos semanas después de la colecta, las semillas de cada una de 10 plantas de las poblaciones Yecorato Mezquite, Texcalama y Alcoyonqui y de seis plantas de la población Yecorato Camino fueron germinadas en condiciones de temperatura fluctuante de 25 a $35{ }^{\circ} \mathrm{C}$ en cámaras de ambiente controlado (Lab Line).

Después de la germinación se tomaron al azar cinco plántulas hijas (una familia) de cada planta y se cultivaron durante $45 \mathrm{~d}$ en charolas de poliestireno, para formar un total de 36 familias. Posteriormente se trasplantaron en 
macetas con $12 \mathrm{~kg}$ de sustrato de 2:1 partes de suelo de aluvión y sustrato organo-mineral ("tierralite"), en un diseño completamente al azar con respecto a la familia y población, en un invernadero bajo malla-sombra que permitió el paso de $50 \%$ de luz solar. En el espacio asignado, las plantas se rotaron al azar cada dos semanas durante los primeros tres meses, y mensualmente en los siguientes cinco meses (Fornoni y Núñez-Farfán, 2002).

Características medidas. Ocho meses después del transplante se midieron las características: altura de planta $(\mathrm{cm})$, diámetro de tallo $(\mathrm{mm})$, largo de hoja $(\mathrm{cm})$, ancho de hoja $(\mathrm{cm})$, número de frutos, número de semillas por fruto, número de semillas por planta y peso individual de semilla (mg). Para obtener el peso de semilla, de cada planta se tomaron al azar 10 frutos y de cada fruto se pesaron 10 semillas individuales en una balanza analítica (Sartorius), con un margen de precisión de $0.1 \mathrm{mg}$.

Análisis estadístico. Se hizo un análisis de varianza anidado para determinar la distribución de las cantidades relativas de variación entre poblaciones, entre familias dentro de población y dentro de familias, para cada característica fenotípica medida. Tanto las poblaciones como las familias dentro de las poblaciones fueron considerados factores aleatorios. Las diferencias entre medias de las poblaciones se determinaron por medio de una prueba de Tukey $(\alpha=0.05)$. Para estimar las contribuciones genéticas en relación al ambiente en la variación de cada característica, se usó la variación dentro de familia como un estimador de la variación ambiental o componente plástico, y la suma de la variación entre población y entre familia como un estimador del componente genético. En plantas que se autofecundan todos los descendientes de un individuo son considerados homocigóticos genéticamente idénticos. Así, la varianza dentro de familia es solamente ambiental, mientras que la varianza entre familia es un componente de la varianza genética total (Venable y Burquez, 1989).

Para cada característica, la varianza dentro de una población se dividió en sus componentes entre familia y dentro familia. Para cada población y característica se estimó la heredabilidad en sentido amplio por medio del coeficiente de correlación intraclase $\left(\mathrm{R}_{\mathrm{I}}\right)$, de acuerdo con Zar (1996). Ri está determinada por la diferencia de las varianzas entre familias y dentro de familias, dividida por su suma. En especies con altos niveles de autofecundación, tales como Capsicum annuum (Pickersgill, 1971b), RI se aproxima al valor de la heredabilidad en sentido amplio. En un diseño con familias de autohermanos, la magnitud de $\mathrm{R}_{\mathrm{I}}$ incluye a los componentes de varianza aditiva y de dominancia; adicionalmente, los efectos maternos también pueden sobreestimar la heredabilidad en sentido amplio, por lo que estos valores pueden ser los máximos estimados (Falconer, 1989). Las diferencias entre poblaciones en las varianzas dentro de familia para cada carácter se probaron estadísticamente con el uso de una prueba de comparaciones múltiples de Levy (Zar, 1996). Todos los análisis se hicieron con el programa estadístico JMP (SAS Institute, 1995).

\section{RESULTADOS Y DISCUSIÓN}

\section{Distribución de la variación}

En todas las características medidas la mayor cantidad relativa de variación se distribuyó dentro de familia (Cuadro 1). Las diferencias dentro de familias explicaron desde $41.8 \%$ (número de frutos por planta) hasta 89.0 (peso individual de semilla) y $89.2 \%$ (número de semillas por fruto) de la variación total, con una media de $61.8 \%$. En cambio, la menor variación se encontró entre poblaciones, con una media de $13.5 \%$ y con un intervalo de variación de 1.5 (peso individual de semilla y número de semillas por fruto) hasta $35.2 \%$ (altura de planta). El promedio de variación entre familias fue $24.7 \%$, con una variación desde 9.3 (número de semillas por fruto) hasta $37.8 \%$ (número de semilla por planta). Estos resultados coinciden con los reportados por Rice y Mack (1991), quienes encontraron que en la estructura de la varianza de número semillas por planta, peso individual de semillas, peso total de semillas y peso seco de planta en Bromus tectorum, fue dominada por la varianza dentro de familia. Esta elevada variación encontrada dentro de familias en las poblaciones de $C$. annuиm silvestre puede ser atribuida al ambiente; sin embargo, debido a que los individuos dentro de la familia no son réplicas de un mismo genotipo (clon), las diferencias genéticas entre los individuos dentro de una familia no se pueden separar de los efectos ambientales.

De la variación fenotípica total observada en todas las características medidas, en promedio $38.2 \%$ tiene una base genética, de la cual $30.1 \%$ se distribuyó entre y $69.9 \%$ dentro de poblaciones (en las familias) (Cuadro 1). Estos resultados indican que las poblaciones silvestres de $C$. annuum del noroeste de México mantienen niveles sustanciales de variación genética estimada en caracteres cuantitativos, y que la mayor parte de esta variación se distribuye dentro de sus poblaciones. Estos resultados coinciden con los publicados por Hernández-Verdugo et al. (2001a) y Oyama et al. (2006), quienes mediante isoenzimas y marcadores moleculares RAPD's mostraron que las poblaciones silvestres de $C$. апnиum del noroeste de México mantienen elevados niveles de variación genética dentro y entre sus poblaciones. Hernández Verdugo et al. (2001a) reportaron que del total de la variación genética estimada con isoenzimas, $94 \%$ se distribuyó dentro y 
$6 \%$ entre las poblaciones. Oyama et al. (2006) estimaron que la variación genética estimada con marcadores moleculares RAPD's se distribuyó equitativamente entre (50 $\%)$ y dentro (50\%) de las poblaciones en $C$. annuum.

\section{Variación entre poblaciones}

Se observaron diferencias significativas entre poblaciones para todas las características (Figura 1). La población Alcoyonqui presentó las plantas con menor altura, con mayor diámetro de tallo y semillas de menor peso (Figura 1A, 1B y 1H). La población Texcalama tuvo las plantas de mayor altura, con menor número de semillas por fruto pero de mayor peso (Figura $1 \mathrm{~A}, 1 \mathrm{~F} \mathrm{y} 1 \mathrm{H}$ ). La población Yecorato Camino tuvo las plantas con mayor ancho de hoja, con menor número de frutos, con mayor numero de semillas por fruto y con menor número de semillas por plantas (Figura 1D, 1E, 1F y 1G). La población Yecorato Mezquite se caracterizó por tener plantas con las hojas menos anchas, con mayor número de frutos y mayor número de semillas por planta (Figura 1D, 1E, y 1G). No obstante que las poblaciones Yecorato Mezquite y Yecorato Camino se encuentran muy cercanas entre sí (500 m de separación), difirieron significativamente en cinco de las ocho características examinadas (Figura 1). Esta elevada diferenciación morfológica observada entre poblaciones cercanas coincide con la reportada por Hernández-Verdugo et al. (1998) para 11 caracteres morfológicos medidos en ocho poblaciones de $C$. annuum silvestre en su ambiente natural.

Estos resultados son consistentes con otros estudios de isoenzimas (Hamrick y Godt, 1997), RAPD's (Bussell, 1999) y caracteres fenotípicos (Rice y Mack, 1991), que han indicado que las especies vegetales con autofertilización muestran diferenciación significativa entre sus poblaciones. La diferenciación entre poblaciones puede ser causada por selección y deriva génica. Sin embargo, no está claro si la variación entre poblaciones en las características estudiadas posee un valor adaptativo. Por ejemplo, la población Yecorato Camino encontrada en un hábitat perturbado a orillas de un camino, con poca vegetación y relativamente altos niveles de luz, presentó las plantas con el mayor largo y ancho de hojas en todas las poblaciones. En cambio, las plantas de la población Yecorato Mezquite que se encontraron creciendo bajo la sombra de mezquites (Prosopis laevigata) mostraron las hojas más pequeñas. Debido a que cantidades bajas de luz restringen la capacidad fotosintética, las plantas expuestas a baja radiación solar responden aumentando el tamaño de hoja para maximizar la intercepción de luz (Sultan y Bazzaz, 1993), por lo que se esperaría que el tamaño de las hojas fuera mayor en la población Yecorato Mezquite. Es posible entonces que la deriva génica sea un factor evolutivo importante en $C$. annuum silvestre.

\section{Variación entre familias}

La heredabilidad en sentido amplio, medida en valores de correlación intraclase, varió entre características y entre poblaciones (Cuadro 2). En todas las características, excepto ancho de hoja y número de semillas por fruto, los valores mínimos de heredabilidad fueron cero o cercanos a cero. Para las características cuyos valores de heredabilidad mínimos fueron cero o cercanos a cero, los valores máximos de heredabilidad variaron de 0.23 (largo de hoja) hasta 0.55 (número de frutos). En ancho de hoja la heredabilidad varió de 0.10 (Yecorato Camino) hasta 0.44 (Yecorato Mezquite). En número de semillas por fruto los valores de heredabilidad estuvieron en un intervalo de 0.39 (Alcoyonqui) hasta 0.75 (Yecorato Mezquite). Las diferencias en la heredabilidad de las características analizadas, sugieren que en el pasado tales características pudieron haber estado sujetas a diferentes presiones de selección en cada población.

Cuadro 1. Porcentaje de variación fenotípica entre poblaciones (VEP), entre familias (VEF) y dentro de familias (VDF). Variación genética total (VGT $=V_{E P}+V_{E F} /\left(V_{E P}+V_{E F}+V_{D F}\right)$, variación genética entre poblaciones $\left(V_{G P}=V_{G P} / V_{G P}+V_{G F}\right) \mathbf{y}$ variación genética entre familias $\left(V_{G F}=V_{G F} /\right.$ $\mathrm{V}_{\mathrm{GP}}+\mathrm{V}_{\mathrm{GF}}$, para ocho caracteres medidos en poblaciones de C. annuum silvestre del Estado de Sinaloa, México.

\begin{tabular}{|c|c|c|c|c|c|c|}
\hline Carácter & $V_{E P}$ & $\mathrm{~V}_{\mathrm{EF}}$ & $\mathrm{V}_{\mathrm{DF}}$ & $\mathrm{V}_{\mathrm{GT}}$ & $\mathrm{V}_{\mathrm{GP}}$ & $\mathrm{V}_{\mathrm{GF}}$ \\
\hline Altura de planta & 35.2 & 22.5 & 42.3 & 57.7 & 61.0 & 39.0 \\
\hline Diámetro de tallo & 13.4 & 30.2 & 56.4 & 43.6 & 30.7 & 69.3 \\
\hline Largo de hoja & 9.5 & 21.6 & 68.9 & 31.1 & 30.5 & 69.5 \\
\hline Ancho de hoja & 10.4 & 29.3 & 60.3 & 39.7 & 26.2 & 73.8 \\
\hline Número de frutos & 21.0 & 37.2 & 41.8 & 58.2 & 36.1 & 63.9 \\
\hline Núm. semillas/fruto & 1.5 & 9.3 & 89.2 & 10.8 & 13.9 & 86.1 \\
\hline Núm. semillas/planta & 15.4 & 37.8 & 46.8 & 53.2 & 28.9 & 71.1 \\
\hline Peso de semilla & 1.5 & 9.6 & 89.0 & 11.4 & 13.2 & 86.8 \\
\hline Media & 13.5 & 24.7 & 61.8 & 38.2 & 30.1 & 69.9 \\
\hline
\end{tabular}



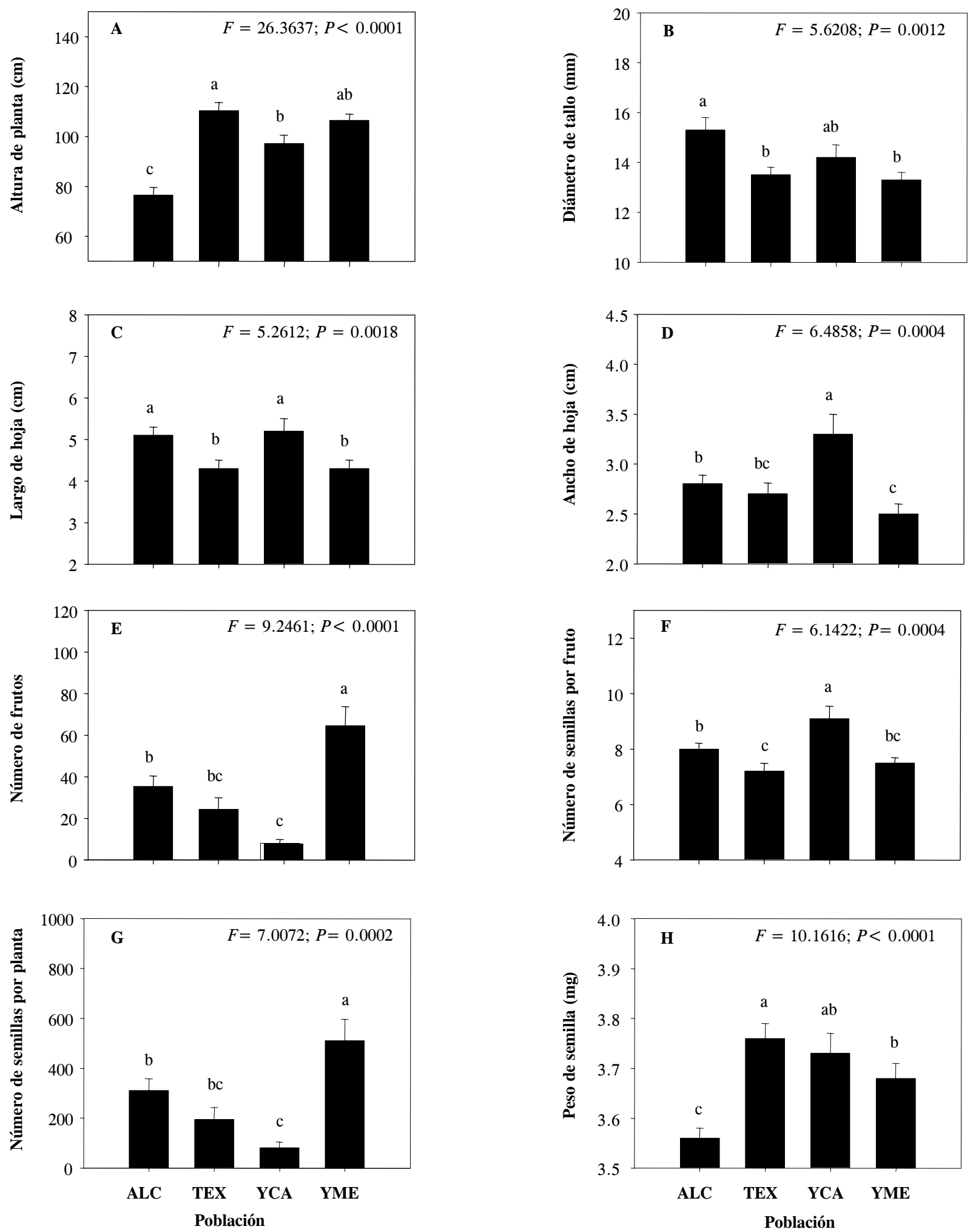

Figura 1. Medias de altura de planta, diámetro de tallo, ancho de hoja, largo de hoja, número de frutos, número de semillas por fruto, número de semillas por planta y peso de semilla, de las poblaciones Alcoyonqui (ALC), Texcalama (TEX), Yecorato Camino (YCA) y Yecorato Mezquite (YME) de $C$. annuum silvestre del Estado de Sinaloa, México. Medias con la misma letra no son estadísticamente diferentes (Tukey, 0.05). 
Los valores de heredabilidad para diámetro de tallo, número de frutos y peso de semilla son consistentes con los reportados por Zhang y Zhou (2006) para estas mismas características en Brassica napus. Estos autores estimaron valores de heredabilidad de $0.22,0.36$ y 0.99 para diámetro de tallo, número de frutos y peso de semilla, respectivamente. La heredabilidad estimada para peso de semilla también fue consistente con el valor de 0.75 estimada para este carácter por Cahill y Ehdaie (2005) en Salvia hispanica. En cambio, Farris (1987) reportó un valor de heredabilidad de cero para esta característica en Cleome serrulata. Los valores de heredabilidad de altura de planta y número de semillas por fruto fueron bajos en relación a los valores de 0.93 y 0.97 obtenidos por Zhang y Zhou (2006) en Brassica napus.

Las diferencias en heredabilidades obtenidas en este estudio para altura de planta, número de semillas por fruto y peso de semilla, en relación con las obtenidas por otros investigadores, pueden ser debido a las diferencias genéticas entre $C$. annuum y las especies por ellos analizadas, así como en los ambientes de evaluación.

En la mayoría las características, la población Yecorato Mezquite presentó heredabilidades mayores que el resto de las poblaciones (Cuadro 2). En las poblaciones Alcoyonqui, Texcalama y Yecorato Camino, tres características diferentes en cada población presentaron valores de heredabilidad de cero o cercanos a cero, con valores máximos de 0.41 (número de semillas por planta), 0.42 (diámetro de tallo) y 0.65 (número de semillas por fruto). En cambio, en la población Yecorato Mezquite las heredabilidades variaron de 0.23 (largo de hoja) a 0.75 (número de semillas por fruto) (Cuadro 2).

Estos resultados sugieren que el potencial para el cambio evolutivo para una característica determinada varía ampliamente entre las poblaciones estudiadas. Las diferencias en la variación potencialmente heredable pueden ocurrir aun a distancias de unos cuantos metros. Por ejemplo, las poblaciones Yecorato Camino y Yecorato Mezquite, separadas por una distancia aproximada de sólo $500 \mathrm{~m}$, difirieron sustancialmente en heredabilidad en sen- tido amplio en cinco de las ocho características, entre ellas dos de las relacionadas directamente con la adecuación: número de semillas por planta y peso de semilla, cuyas heredabilidades fueron 0.55 , y 0.36 en la población Yecorato Mezquite, y de 0.12 y 0.07 en la población Yecorato Camino.

Los valores bajos de heredabilidad de la población Yecorato Camino en relación con la población Yecorato Mezquite, pueden ser producto del tamaño pequeño de la población. Sólo se formaron seis familias de la población Yecorato Camino, porque ése fue el número de plantas que se encontraron con frutos suficientes para los experimentos. Se ha estimado que cuando una población experimenta un "cuello de botella" genético, con un número de individuos menor que 10 , se reduce significativamente el número de alelos por locus (Nei et al., 1975). Estas diferencias en las cantidades de variación genética entre poblaciones y características pueden tener impacto tanto en la tasa como en la dirección del cambio evolutivo en estas poblaciones de chile silvestre del noroeste de México.

\section{Variación dentro de familias}

El porcentaje de la variación dentro de familias fue alto y se distribuyó de manera homogénea entre poblaciones en todas las características, excepto en diámetro de tallo (Cuadro 3). Las características peso de semilla y número de semillas por fruto fueron igual o mayor a $80 \%$ en todas las poblaciones; diámetro de tallo y largo de hoja solamente superaron el $80 \%$ en la población Alcoyonqui. Sólo la característica diámetro de tallo fue inferir a $50 \%$ en la población Texcalama. Esta elevada variación dentro de las familias coincide con la observada por Rice y Mack (1991), quienes reportaron que la varianza dentro de familia fue el componente dominante para número de semillas por planta, peso individual de semilla, peso total de semillas y peso seco de planta. En Bromus tectorum Rice y Mack (1991) consideraron que la elevada varianza dentro de familias era producida por diferencias en la capacidad de responder al ambiente, conocida como plasticidad fenotípica, debido a la casi homocigosis completa.

Cuadro 2. Valores de heredabilidad en sentido amplio medidos en coeficientes de correlación intraclase (R⿺) para las características altura de planta (APL), diámetro de tallo (DTA), largo de hoja (LHO), ancho de hoja (AHO), número de frutos (NFR), número de semillas por fruto (NSF), número de semillas por planta (NSP) y peso de semilla (PSE) en poblaciones de C. annuum silvestre del Estado de Sinaloa, México.

\begin{tabular}{|c|c|c|c|c|c|c|c|c|}
\hline \multirow{2}{*}{ Población } & \multicolumn{8}{|c|}{ Carácter } \\
\hline & APL & DTA & LHO & AHO & NFR & NSF & NSP & PSE \\
\hline Alcoyonqui & 0.36 & 0.00 & 0.00 & 0.17 & 0.28 & 0.39 & 0.41 & 0.00 \\
\hline Texcalama & 0.38 & 0.42 & 0.22 & 0.13 & 0.00 & 0.41 & 0.01 & 0.03 \\
\hline Yecorato Camino & 0.00 & 0.31 & 0.23 & 0.10 & 0.04 & 0.65 & 0.12 & 0.07 \\
\hline Yecorato Mezquite & 0.26 & 0.34 & 0.23 & 0.44 & 0.55 & 0.75 & 0.55 & 0.36 \\
\hline
\end{tabular}


Cuadro 3. Porcentaje de variación dentro de familias para ocho caracteres estudiados en las cuatro poblaciones de chile silvestre $(C$. annuum) colectadas en el Estado de Sinaloa, México.

\begin{tabular}{|c|c|c|c|c|c|c|c|c|}
\hline \multirow{2}{*}{ Población } & \multicolumn{8}{|c|}{ Carácter } \\
\hline & APL & DTA & LHO & $\mathrm{AHO}$ & NFR & NSF & NSP & PSE \\
\hline Alcoyonqui & $61.2 \mathrm{a}$ & $85.2 \mathrm{a}$ & $88.8 \mathrm{a}$ & $70.8 \mathrm{a}$ & $62.5 \mathrm{a}$ & $80.3 \mathrm{a}$ & $56.8 \mathrm{a}$ & $80.5 \mathrm{a}$ \\
\hline Texcalama & $51.1 \mathrm{a}$ & $48.2 \mathrm{~b}$ & $59.7 \mathrm{a}$ & $64.2 \mathrm{a}$ & $65.6 \mathrm{a}$ & $89.4 \mathrm{a}$ & $62.8 \mathrm{a}$ & $90.0 \mathrm{a}$ \\
\hline $\begin{array}{l}\text { Yecorato } \\
\text { Camino }\end{array}$ & $77.4 \mathrm{a}$ & $62.3 \mathrm{ab}$ & $67.1 \mathrm{a}$ & $72.3 \mathrm{a}$ & $67.6 \mathrm{a}$ & $89.4 \mathrm{a}$ & $63.7 \mathrm{a}$ & $95.5 \mathrm{a}$ \\
\hline $\begin{array}{l}\text { Yecorato } \\
\text { Mezquite }\end{array}$ & $68.2 \mathrm{a}$ & $64.0 \mathrm{ab}$ & $69.7 \mathrm{a}$ & $58.7 \mathrm{a}$ & $49.1 \mathrm{a}$ & $91.7 \mathrm{a}$ & $50.1 \mathrm{a}$ & $80.7 \mathrm{a}$ \\
\hline
\end{tabular}

Medias con letras iguales en cada columna no son estadísticamente diferentes (Levy, 0.05). Las abreviaturas de los caracteres son las mismas que en el Cuadro 2.

La alta variación dentro de familias mostrada en las poblaciones de $C$. annuum silvestre puede ser producida por diferencias en la sensibilidad al ambiente o plasticidad fenotípica de los individuos dentro de las familias. Sin embargo, debido a que los genotipos dentro de las familias no se replicaron (clonaron), las diferencias genéticas entre los individuos no se pueden separar de los efectos del ambiente. Estudios anteriores con isoenzimas (Hernández-Verdugo et al., 2001a) mostraron que estas mismas poblaciones de $C$. annuum silvestre mantienen altos niveles de heterocigosis. Esta alta plasticidad fenotípica, junto con la variación genética dentro y entre las poblaciones de chile silvestre, puede contribuir a que $C$. annuum silvestre alcance el amplio rango de distribución geográfica en el continente americano, desde el sur de los Estados Unidos hasta el norte de Argentina (Pickersgill 1971a; D'Arcy y Eshbaugh, 1974).

\section{CONCLUSIONES}

Las poblaciones de Capsicum annuum silvestre mantienen relativamente altos niveles de variación fenotípica dentro y entre sus poblaciones. Las poblaciones se diferenciaron significativamente en todas las características medidas. De la variación fenotípica total, en promedio $61.8 \%$ se encontró dentro de familias, $24.7 \%$ entre familias y $13.5 \%$ entre poblaciones. De la variación fenotípica total observada, en promedio $38.2 \%$ tuvo una base genética. De esta proporción de variación genética, 69.9 $\%$ se distribuyó dentro de poblaciones (entre familias) y $30.1 \%$ entre poblaciones. Esto significa que las poblaciones de $C$. апnиum silvestre mantienen relativamente altos niveles de variación genética dentro y entre sus poblaciones. La heredabilidad en sentido amplio $\left(R_{1}\right)$ varió entre características y entre poblaciones. Seis de las ocho características presentaron valores de heredabilidad mínimos de cero o cercanos a cero en al menos una población, con valores máximos de 0.26 a 0.55 . En las otras dos características la heredabilidad varió de 0.10 a 0.75 . Esto sugiere que tales características estuvieron sometidas a diferentes presiones de selección en el pasado, con diferentes picos adaptativos en cada población.

\section{AGRADECIMIENTOS}

A la Universidad autónoma de Sinaloa por el apoyo financiero (proyecto PROFAPI 2007/50), al CONACYT (proyecto S56261-Z), al CONACYT-Gobierno del Estado de Sinaloa (FOMIX proyecto 71381) otorgado a Sergio Hernández Verdugo. A Heriberto Luna Barraza y Faridh A. Lerma Santiago, por su asistencia en el trabajo de campo y laboratorio.

\section{BIBLIOGRAFÍA}

Bussell D (1999) The distribution of random amplified polymorphic DNA (RAPD) diversity amongst populations of Isotoma petreae (Lobeliaceae). Mol. Ecol. 88:775-789.

Cahill J P, B Ehdaie (2005) Variation and heritability of seed mass in chia (Salvia hispanica L.). Gen. Res. Crop Evol. 52:201207.

D'Arcy W G, W H Eshbaugh (1974) New World peppers (CapsicumSolanaceae) north of Colombia. Baileya 19:93-103.

Falconer D S (1989) Introduction to Quantitative Genetics. 3rd ed. Longman Scientific \& Technical. London UK. 438 p.

Farris M A (1987) Quantitative genetic variation and natural selection in Cleome serrulata growing along a mild moisture gradient. Can. J. Bot. 66:1870-1876.

Felde T, H C Becker, C Möllers (2006) Genotype x environment interactions, heritability, and traits correlations of sinapate ester content in winter rapeseed (Brassica napus L.). Crop Sci. 46:2195-2199.

Fornoni J, J Núnez-Farfán (2002) Evolutionary ecology of Datura stramonium: genetic variation and costs for tolerance to defoliation. Evolution 54:789-797.

Hamrick J T, M J W Godt (1997) Allozyme diversity in cultivated crops. Crop Sci. 37:26-30.

Hernández Verdugo S, R G Guevara González, R F Rivera Bustamante, C Vázquez Yanes, K Oyama K (1998) Los parientes silvestres del chile (Capsicum spp.) como recursos genéticos. Bol. Soc. Bot. Méx. 62:171-181.

Hernández Verdugo S, P Dávila, K Oyama (1999) Síntesis del conocimiento taxonómico, origen y domesticación del género Capsicum. Bol. Soc. Bot. Méx. 64:65- 84.

Hernández Verdugo S, R Luna-Reyes, K Oyama (2001a) Genetic structure and differentiation of wild and domesticated populations of Capsicum annuum from Mexico. Plant Syst. Evol. 226:129-142.

Hernández Verdugo S, K Oyama, C Vázquez Yanes (2001b) Differentiation in seed germination among populations of Capsicum anпиum along a latitudinal gradient in Mexico. Plant Ecol. 155:245-257.

Hernández Verdugo S, R G Guevara González, R F Rivera Bustamante, K Oyama (2001c) Screening wild plants of Capsicum 
аппиит for resistance to pepper huasteco virus: presence of viral DNA and differentiation among populations. Euphytica 122:31-36.

Hernández Verdugo S, A González Rodríguez, P Sánchez Peña, A Casas, K Oyama (2006) Estructura y diferenciación genética de poblaciones silvestres y domesticadas de chile del noroeste de México analizadas con isoenzimas y RAPDs. Rev. Fitotec. Mex. 29 (Núm. Especial 2):25-29.

Kimura M, T Otha (1971) Theoretical Aspects of Population Genetics. Princeton University Press. Princenton, New Jersey, USA. $219 \mathrm{p}$.

Nei M, T Maruyama, R Chakraborty (1975) The bottleneck effect and genetic variability in populations. Evolution 29:1-10.

Oyama K, S Hernández-Verdugo, C Sánchez, A González-Rodríguez, P Sánchez-Peña, J A

Garzón-Tiznado, A Casas (2006) Genetic structure of wild and domesticated populations of Capsicum annuum (Solanaceae) from northwestern Mexico analized by RAPDs. Gen. Res. Crop Evol. 53:553-562.

Pickersgill B (1971a) Relationships between weedy and cultivated forms in some species of chili peppers (genus Capsicum). Evolution 25:683-691.

Pickersgill B (1971b) Cytogenetics and evolution of Capsicum L. In: Chromosome Engineering in Plants: Genetics, Breeding, Evolution. Part B. T Tsuciya, P K Gupta (eds). Elsevier. Oxford, UK. pp:139-160.
Rice K J, R N Mack (1991) Ecological genetics of Bromus tectorum. I. A hierachical analysis of phenotypic variation. Oecologia 88:77-83.

SAS Institute (1995) Statistical and Graphical Guide. SAS Institute Inc. Cary, North Caroline.

Sultan S E, F A Bazzaz (1993) Phenotypic plasticiy in Polygonum persicaria. I. Diversity and uniformity in genotypic norms of reaction to light. Evolution 47:1009-1031.

Vázquez Dávila M A (1996) El amash y el pistoqué: un ejemplo de la etnoecología de Los Chontales de Tabasco, México. Etnoecológica 3:59-69.

Venable D L, A M Burquez (1989) Quantitative genetics of size, shape, life-history, and fruit characteristics of the seedheteromorphic composite Heterosperma pinnatum. Evolution 43:113-124.

Vida G (1994) Global issues of genetic diversity. In: Conservation Genetics. V Loeschcka, J Tomiuk, S K Jain (eds). Bikhäuser Verlag. Berlin, Germany. pp:9-19.

Wright $S$ (1951) The genetic structure of populations. Ann. Eugenesic 15:323-354.

Zar J H (1996) Biostatistical Analysis. 3rd ed. Prentice Hall. New Jersey, USA. $662 \mathrm{p}$.

Zhang G, W Zhou (2006) Genetics analysis of agronomic and seed quality traits of synthetic oilseed Brassica napus produced from interspecific hybridization of $B$. campestri and $B . o l$ eracea. J. Genetics 85:45-51. 